TAO, Vol. 17, No. 1, 263-276, March 2006

\title{
Ionospheric Tomography from a Reference GPS/MET Experiment Through the IRI Model
}

\author{
Lung-Chih Tsai ${ }^{1,2, *}$, Wei-Hsiung Tsai ${ }^{2}$, Jan-Yin Chou ${ }^{2}$, and Chao-Han Liư ${ }^{2}$
}

(Manuscript received 28 July 2004, in final form 3 October 2005)

\begin{abstract}
In earlier studies, we implemented the Multiplicative Algebraic Reconstruction Technique (MART) to reconstruct two-dimensional ionospheric structures from measured TECs through the receptions of the GPSto-LEO signals and/or the NNSS-to-ground beacon signals. To examine the accuracy of the reconstructed image we need ground-based validation systems which are difficult to obtain. However, such comparative investigation is needed if one aims to improve tomography inverse techniques and algorithms. In this study, we propose a simulation scheme to carry out this task. We first simulate the GPS-to-LEO TEC measurements through the IRI model by integrating electron densities along the "straight" ray occultation paths between the GPS and LEO satellite obtained from the real GPS/MET experiment. Contiguous tomographic images are then derived by the MART algorithm within the "reference" GPS/MET experiment. They are verified by comparison with the "true" IRI-modelled ionosphere. We show that simulation/reference results can be used to find the optimal reconstruction strategy in space-based ionospheric tomography.
\end{abstract}

(Key words: Ionospheric tomography, Total electron content (TEC), the Multiplicative Algebraic Reconstruction Technique (MART), GPS occultation)

\section{INTRODUCTION}

Tomography has been applied to many different fields (e.g., see Lee and Wade 1985). Its

\footnotetext{
${ }^{1}$ Center for Space and Remote Sensing Research, National Central University, Chung-Li, Taiwan, ROC

2 Institute of Space Science, National Central University, Chung-Li, Taiwan, ROC

* Corresponding author address: Prof. Lung-Chih Tsai, Center for Space and Remote Sensing Research, National Central University, Chung-Li, Taiwan, ROC; E-mail: Ictsai@csrsr.ncu.edu.tw
} 
application to ionospheric research was first proposed by Austen et al. $(1986,1988)$ to image the electron density distribution in the ionosphere. From earlier studies (Austen et al. 1988; Huang et al. 1997, 1999; Raymund et al. 1990, 1994; Sutton and Na 1995) it has been established that Computerized Ionospheric Tomography (CIT) can provide information to resolve both horizontal and vertical structures of electron density distribution over large geographic coverage. These experiments of ionospheric tomography were conducted using Total Electron Contents (TECs) derived from satellite-beacon signals received at ground-based stations. Due to the geometry of the propagation paths, the reconstructed electron density distribution can provide detailed information on the horizontal structure but is of restricted utility in sensing vertical structure. Obviously, it is also limited to a local area depending on the distribution of ground-based receivers.

In 1993 the University Corporation for Atmospheric Research (UCAR) conducted a proofof-concept experiment on a LEO satellite (the MicroLab-1 satellite) at a nearly circular orbit of $\sim 735 \mathrm{~km}$ altitude and $\sim 70^{\circ}$ inclination angle to receive mutli-channel GPS carrier phase signals (1.5 GHz and 1.2 GHz). From April of 1995 to February of 1997, an occultation observation mission termed the Global Positioning System/Meteorology (GPS/MET) program demonstrated active limb sounding of the Earth's atmosphere and ionosphere and collected several tens of thousands of occultation observations. The main objectives of this GPS-LEO occultation mission were numerical weather prediction and long-term monitoring of the Earth's climate (Zou et al. 1995; Ware et al. 1996; Kursinski et al. 1997). GPS/MET has also been able to sound the ionosphere from the orbit altitude to the Earth's surface and retrieve one-dimensional profiles of ionospheric electron density using the Abel integral transform under the assumption of spherical symmetry at the locality of occultation. There are several studies to examine and estimate the accuracy of retrieved GPS/MET electron density profiles obtained with the Abel inversion by comparing them with vertical ionosonde data (Hajj and Romans 1998; Schreiner et al. 1999; Tsai et al. 2001; Tsai et al. 2004). Meanwhile, two-dimensional tomographic reconstruction from GPS/MET data have been demonstrated by Rius et al. (1997, and 1998), Hajj et al. (2000), and Tsai et al. (2002). In our earlier works (Tsai et al. 2002) we have implemented the Multiplicative Algebraic Reconstruction Technique (MART) to reconstruct the ionosphere projected onto the LEO-orbital plane using measured TECs through the receptions of the GPS-to-LEO signals and/or the NNSS-to-ground signals. The reconstructed electron density variations seem to show better results than the original vertical profiles retrieved from the GPS occultation data by the Abel transformation, being in better agreement in peak electron density to nearby ionosonde measurements. Obviously, more need be done to improve tomography inverse techniques and algorithm. However, validations of the inversion results require data from ionosondes and incoherent scatter radars located near the reconstruction plane. Unfortunately, actual GPS/MET data can only provide a limited number of such coincidental cases. Therefore, a comprehensive investigation of the reconstruction algorithm is difficult.

In this paper, we propose to solve this problem using simulated data obtained from a known ionosphere, namely, the International Reference Ionosphere (IRI) model. The IRI model has been developed for more than 30 years and achieved quite a realistic description of the highly variable ionosphere (Bilitza 2001). The simulated path TECs used in the inversion are 
calculated by integrating electron density under the IRI model along the known paths obtained from the actual GPS/MET mission. Under the assumption of straight-line propagation, different combinations of simulated paths TECs from occultation observations within each one, two, and four revolutions of the MicroLab-1 satellite around the Earth have been used for different tomographic reconstructions of the ionosphere. The reconstructed ionospheric maps are compared with the "true" ionosphere from the IRI model.

\section{GPS-LEO RADIO OCCULTATION OBSERVATIONS AND THE GEOMETRY FOR IONOSPHERIC TOMOGRAPHY}

As shown in Fig. 1 of a GPS-LEO geometrical optics approximation, an occultation takes place when a GPS satellite sets or rises behind the Earth's ionosphere and lower neutral atmosphere as seen by a receiver in LEO. Each occultation therefore consists of a set of limbviewing links with ray perigee ranging from the LEO satellite height to the surface of the Earth. A ray passing through the ionosphere and the neutral atmosphere is actually refracted according to Snell's law due to the vertical gradient of refractive index. The overall effect of the refractive index changes can be used to extract information such as profiles of electron density in the ionosphere, or temperature, pressure and water vapor in the lower neutral atmosphere. However, even during the daytime and near solar maximum, the absolute magnitude of the bending angle for the GPS rays propagating through the ionosphere above the Eregion does not exceed $0.016^{\circ}$ and $0.026^{\circ}$ for $\mathrm{L} 1(1.57542 \mathrm{GHz})$ and $\mathrm{L} 2(1.22760 \mathrm{GHz}) \mathrm{GPS}$ frequencies, respectively (Hajj and Romans 1998; Schreiner et al. 1999). We can therefore safely assume that for TEC calculations all rays are considered as straight lines from a GPS

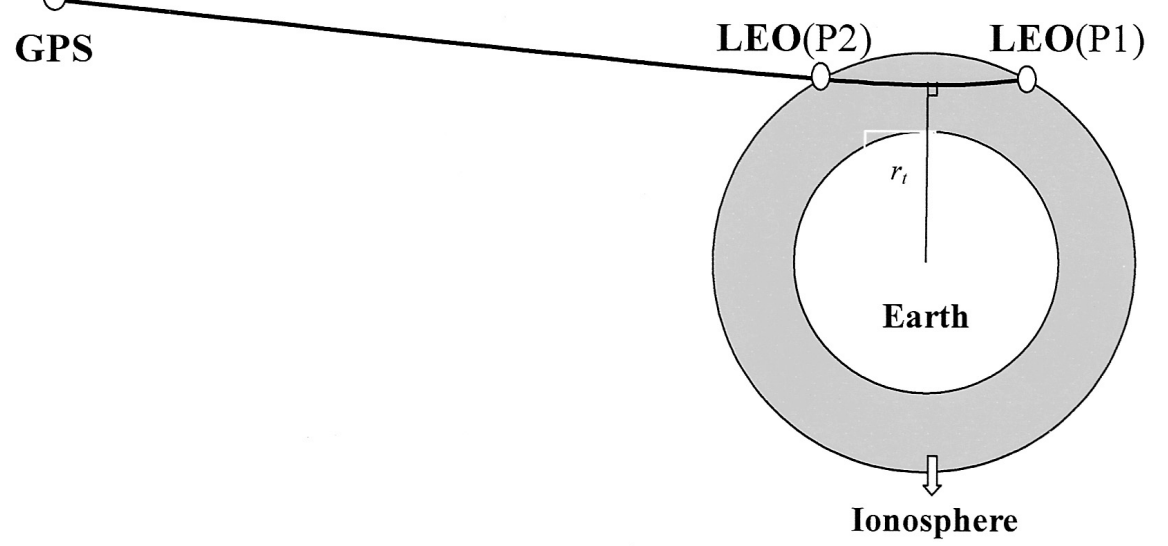

Fig. 1. Illustration of the geometry of the GPS-LEO occultation problem for ionosphere observations, where $\mathrm{P} 1$ is an occulting LEO point, and $\mathrm{P} 2$ is an auxiliary LEO point with the same impact distance of P1. 
transmitter to a receiver in LEO. This simplifies the corresponding radio ray geometry problem for CIT inversion.

An example of a data scenario for tomographic reconstruction using occultation observations is shown in Fig. 2. We discretize the reconstruction plane, i.e., the MicroLab-1 orbital plane at $\sim 70^{\circ}$ inclination, into radial pixels. To illustrate the reconstruction approach limb links from different occultation observations have been shown in different colors and onto the LEO-orbital plane. In the GPS/MET mission, the Microlab-1 spacecraft was equipped with a single antenna that tracked the occulted GPS satellites and the occultations may not be exactly in the LEO orbital plane. In fact we have to assume a certain extent of the tomography pixels (perpendicular to the reconstruction plane) to be "three-dimensional" cells to cover most of the out-of-plane paths between the occulting LEO positions and their corresponding calibrated positions from several occultations (Tsai et al. 2002). In the actual GPS/MET mission, a GPS receiver onboard the MicroLab-1 satellite can provide up to 250 useful occultations per day based on 24 GPS satellites and $60^{\circ}$ viewing angle, i.e., $\sim 17$ occultation observations during one revolution of the MicroLab-1 satellite. Meanwhile, the MicroLab-1 LEO orbit estimation has been performed with GPS triple difference measurements at a rate of $0.1 \mathrm{~Hz}$ and can

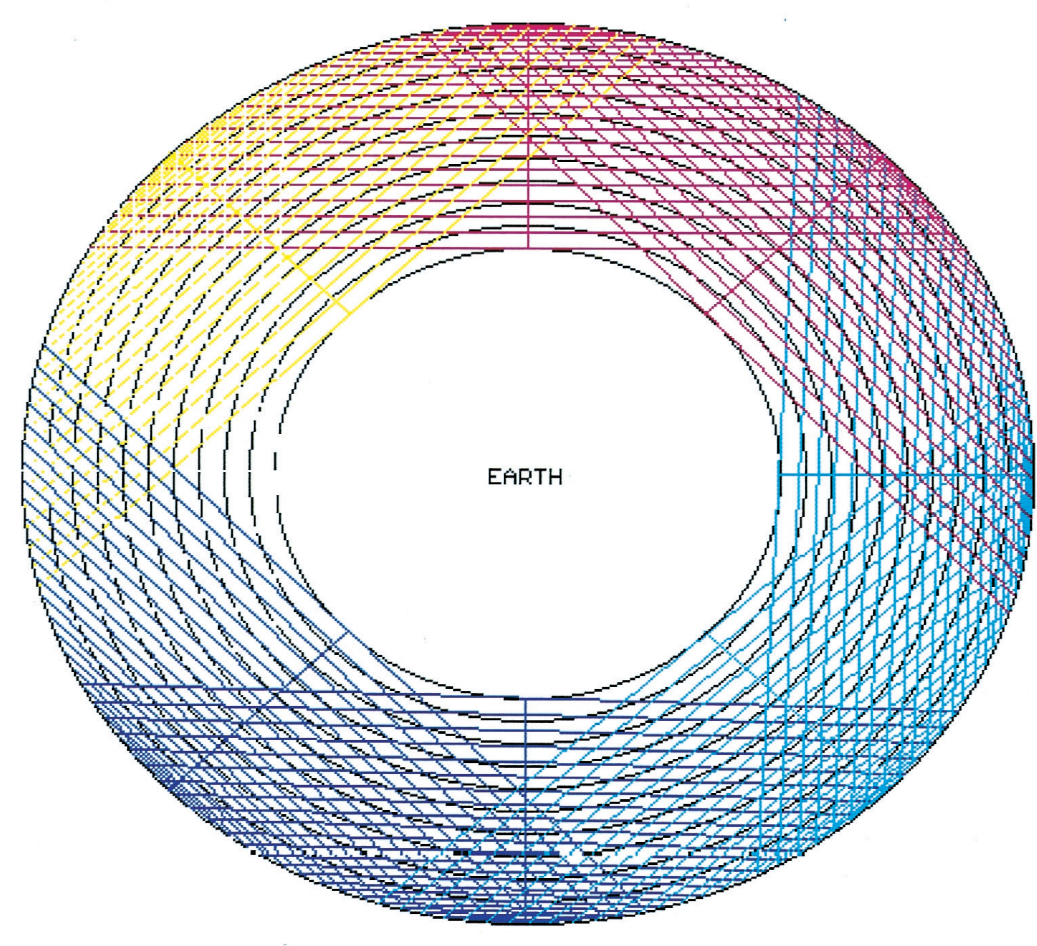

Fig. 2. Example of occultation data scenario for ionospheric tomography reconstruction. 
approach a position precision of $\sim 15 \mathrm{~cm}$ 3D root mean square (Schreiner et al. 1998). In the reference GPS/MET experiment, we use the precisely determined orbit information for the MicroLab-1 satellite to simulate the path TEC values by integrating the electron densities along "straight" paths through the IRI model corresponding to the real GPS/MET experiment. The simulated TEC values are calculated by numerical integration using Gaussian Quadrature of order 7 over an interval of $50 \mathrm{~km}$ along straight paths between the occulting LEO positions and their associated "calibrated" positions having the same impact distance as illustrated as the P1 and P2 in Fig. 1. The calculated $0.1 \mathrm{~Hz}$ TEC values are, however, interpolated to 300 values from top to bottom of occultation using a cubic spline fit. Under the assumption of straight-line propagation, in each occultation observation a set of 300 path TECs can be determined and used for tomographic reconstruction of the ionosphere region below the LEO orbital height.

\section{TEC SIMULATIONS USING THE IRI}

The International Reference Ionosphere (IRI) model (Rawer et al. 1978, 1978, 1981; Bilitza $1990,2001)$ is not a theoretical model but an empirical standard ionosphere model based on many data sources including the worldwide network of more than one hundred ionosondes, the powerful incoherent scatter radars (Jicamarca, arecibo, Millstone Hill, Malvern, St. Santin), the ISIS and Alouette topside sounders, and in situ instruments on several satellites and rockets. In this paper, we use the IRI-2001 model of monthly averages in the non-auroral ionosphere for magnetically quiet conditions. Other extensions of disturbed ionosphere can be planned under the conditions of the ion drift model, description of the auroral and polar ionosphere, and magnetic storm effects. To present the ionosphere based on the IRI, Fig. 3 shows four calculated 100-minute electron density images onto the LEO-orbital-plane from 02:40 to 09:00 UT on the $8^{\text {th }}$ of February in 1997. The ionospheric images are indicated by altitudes between 0 to $800 \mathrm{~km}$ and plane latitudes, where the zero degree corresponds to the equator, the left-half part from $-90^{\circ}$ to $90^{\circ}$ presents dayside electron densities, and the right-half part from $90^{\circ}$ to $-90^{\circ}$ presents nightside electron densities. We note that, in the dayside equatorial and low-latitude region of Fig. 3, features of the equatorial anomaly were captured in the IRI model, and the two crests lie at about $+25^{\circ}$ and $-5^{\circ}$ LEO plane latitudes $\left(\sim 15^{\circ}\right.$ and $-15^{\circ}$ geomagnetic latitudes, respectively).

\section{THE MART ALGORITHM FOR TOMOGRAPHIC RECONSTRUCTION}

To illustrate the approach of tomographic reconstruction we firstly choose to combine occultations taking place during one orbital revolution of the Microlab-1 satellite within the GPS/MET mission. The tomography geometry onto the LEO orbital plane (at $70^{\circ}$ inclination angle) is built at a cell resolution of $0.25^{\circ}$ in latitude and $10 \mathrm{~km}$ in altitude which are about one order greater than the $2 \mathrm{~km}$ theoretical vertical resolution limited by diffraction effects. With the straight-line propagation assumption, Fig. 4 shows the side view and log information of the limb-viewing links of 20 occultation observations obtained during one pass of the Microlab- 

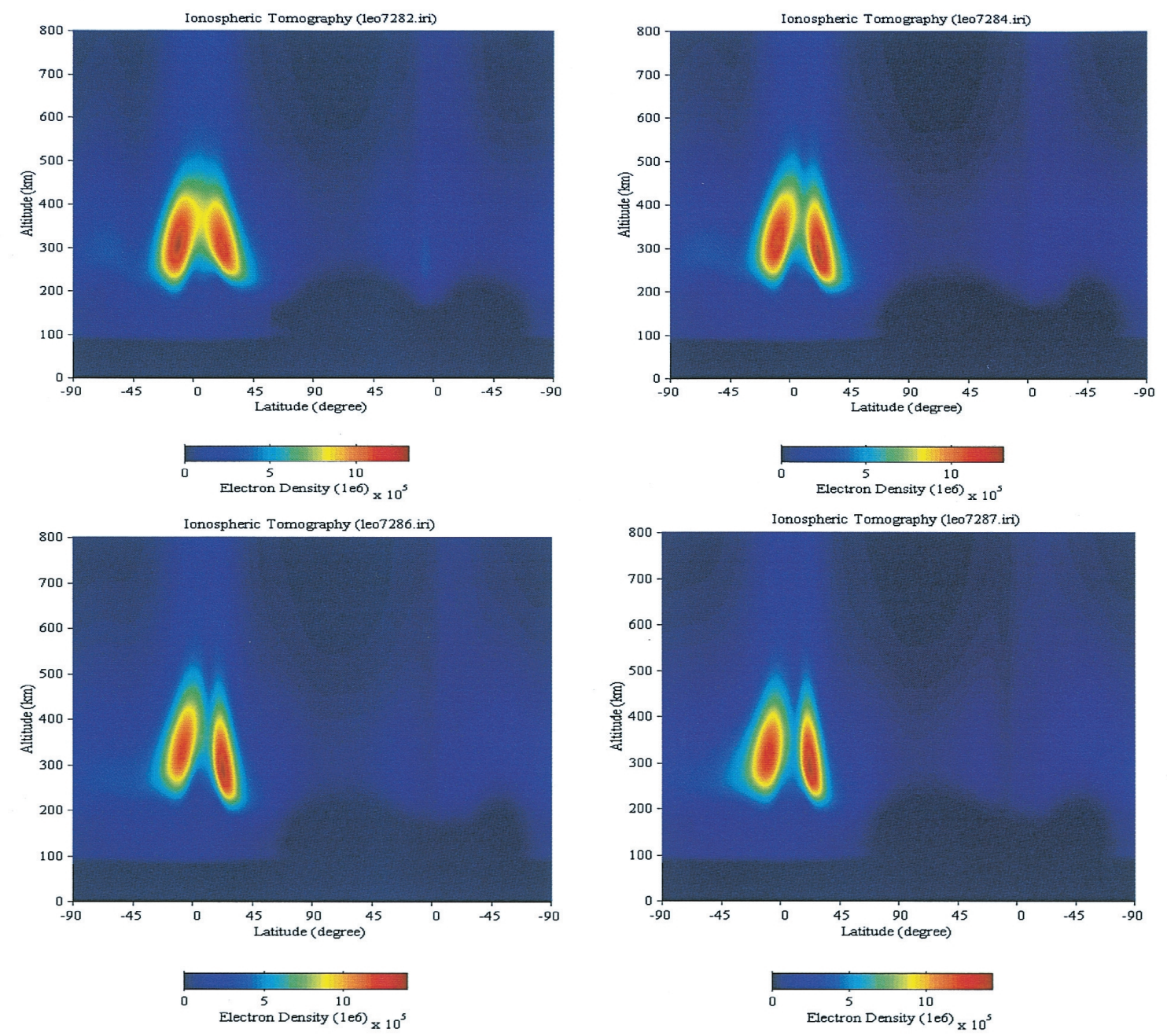

Fig. 3. Four 100-minute electron density images during 0200 to 0900 UT on Feb. 8, 1997, to be true ionosphere based on the IRI model and used to verify the reconstructed tomography produced within the reference GPS/ MET experiment.

1 orbit from 02:40 to 04:15 UT on Feb. 8, 1997. We note the sparse coverage of GPS/MET onto the reconstruction plane. The path TEC values for these GPS-LEO occultations can be expressed as an algebraic equation in matrix form:

$$
Y=A X+E,
$$




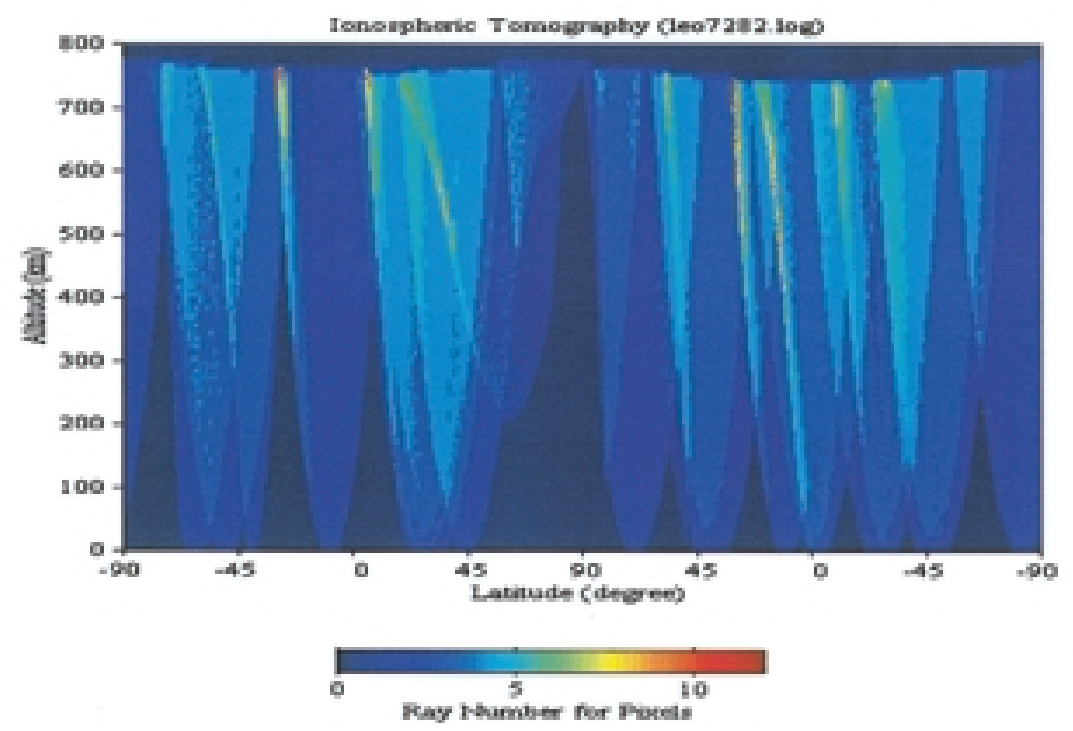

Fig. 4. The side view and log information of occultation links taking place during 02:00 to 04:00 UT on Feb. 8, 1997, within the GPS/MET mission.

where $Y$ is a column of $m$ TEC records; $X$ is a stacked vector of $n$ electron density unknowns for discrete cells in the targeted two-dimensional ionosphere region and is assumed to be constant inside each cell. Depending on the geometry basis, $A$ is an $m \times n$ observation matrix with $a_{j i}$ being the link length of the jth ray $(1 \leq j \leq m)$ that lies in cell $\mathrm{i}(1 \leq i \leq n)$, and $\mathrm{E}$ represents TEC record errors. In this study, the "recorded" TECs are simulated by the line integrations of the IRI modelled electron densities along calibrated paths, the link length $a_{j i}$ can be determined with excellent accuracy from precise orbit estimations during the actual GPS/MET experiment, and the contents of $E$ are mostly caused by numerical calculation errors and can be ignored here.

Because of the sparse geometry coverage the tomography inverse problem as described is underdetermined for solving a system of linear equations. Given that the data are incomplete, there are many possible solutions to this system. Raymund (1994) reviewed Computerized Ionospheric Tomography reconstruction algorithms proposed by various investigators. One of the most commonly used approaches is the Algebraic Reconstruction Technique (ART) first introduced in CIT by Austen et al. (1988). The ART algorithm solves the inverse problem by iteratively adding improvements to an initial guess of $X$. A modified version of ART is the socalled multiplicative ART (MART) in which the correction of X in each iteration is obtained by making a multiplicative modification rather than an additive correction (Raymund et al. 1990). The iterative algorithms ART and MART are appealing because they prove to be more robust in cases with incomplete data and they use less computer memory and require less computation. 
MART has an advantage over ART in that it can avoid unreasonable negative values for electron density in the reconstruction. Basically, the MART algorithm is taken up cyclically and can be implemented as the following iterative equation:

$$
x_{i}^{k+1}=\left[\frac{y_{i}}{\left(a^{j}, x^{k}\right)}\right]^{\lambda_{k} a_{i}^{j}} \cdot x_{i}^{k} \quad, \quad i=1,2, \perp, n,
$$

where $x_{i}$ is the electron density for the $i$ th resulted cell in a column of $n$ unknowns of $X$. In the iteration, it is corrected by a multiplication factor as shown, where $y_{j}$ is the jth recorded TEC in a $m$-element vector of $Y, a_{i}^{j}$ being the length of link $j$ that lies in cell $i$, and the inner product of the vectors $X^{k}$ and $a^{j}$ is thus the revised TEC value for the link $j$ at the kth iteration. Furthermore, $\lambda_{k}$ is a relaxation parameter at the kth iteration and $0<\lambda_{k}<1$. We continue by calculating the revised path TECs within the determined pre-cycle electron density image and the ratios of the revised path TECs to the recorded path TECs (originally simulated through the IRI model within the reference GPS/MET experiment) for each iteration; the electron density matrix $\mathrm{X}$ is therefore corrected in iteration by a ratio of the recorded TECs and the revised TECs with a relaxation parameter of $\lambda$. The MART algorithm is usually iterated $\sim 10$ times depending on the choice of the relaxation parameters. In practice, the MART algorithm often uses a series of decreasing relaxation parameters so that a more correct electron density image is modified less as the revised TECs approach the originally recorded TECs.

It is noted that the MART algorithm, as with other iterative techniques, requires an initial condition before iteration begins. For cases where available data are incomplete due to propagation geometry such as the limited occultation observations available within the GPS/MET experiment, the initialization image could dominate the reconstruction. Earlier, ionospheric tomography algorithms used a priori information such as ground-based observations or the IRI models (Raymund et al. 1994; Huang et al. 1997) to minimize the effects of incomplete data. In this study we use the ionospheric density profiles derived from the occultation observations as our initial guess. We note that the retrieval of one-dimensional ionospheric profiles generally assumes spherical symmetry of electron density and uses the Abel integral transform to invert TEC data (or bending angle) to one-dimensional electron density profiles. For the GPS/MET operation, approximately 15 occultation observations during one revolution of the MicroLab-1 satellite around the Earth can be successfully used to retrieve vertical electron density profile through the Abel transformation. A two-dimensional electron density distribution can thus be deduced through relaxation using red-black smoothing. This two-dimensional ionosphere image is used as the initial guess to start the MART algorithm. Actually, the vertical electron density profiles derived by the Abel inversion approach have been examined by comparing the determined foF 2 and $h m F 2$ values with vertical ionosonde data (Hajj and Romans 1998; Schreiner et al. 1999; Hajj et al. 2000; Tsai et al. 2001). All of the works obtained fractional $r m s$ differences from $20 \%$ to $40 \%$ in NmF2 implying differences from $10 \%$ to $20 \%$ in $f o F 2$. Such initial approximation of red-black smoothing on the Abel inversion derived global profiles will be improved as we go through the tomographic inversion. 


\section{TOMOGRAPHY RESULTS OF THE REFERENCE GPS/MET EXPERIMENT}

Because of the lack of occultation observational data in the GPS/MET experiment, we also collected a sufficient amount of occultation data within several (NOT only one) LEO satellite revolutions for the tomographic reconstruction. Thus, we trade off temporal and/or spatial resolutions. The mapping covers the region onto the MicroLab-1 orbital plane including both day- and night-sides of the ionosphere. Three cases of different combinations of simulated path-TECs from occultation observations within one, two, and four revolutions of the MicroLab-1 satellite around the Earth have been used for tomographic reconstructions. These correspond to $\sim 17, \sim 34$, and $\sim 68$ occultation observations, respectively. The electron profiles derived from the simulated GPS/MET occultation data for that same period are used as initial guess for the MART reconstruction. We have evaluated the performance of the tomographically derived ionosphere by comparing it with the "true" IRI-modelled ionosphere as shown in the examples of Fig. 3, where features of the equatorial anomaly were captured, and the two crests lay at about $+22^{\circ}$ and $-5^{\circ}$ LEO plane latitudes. Furthermore, as shown in these four true ionospheric images of Fig. 3, the south-crest peak electron densities were about the same as the north-crest peak densities at about 12.5 units (each unit is $10^{11} \mathrm{el} \mathrm{m}^{-3}$ ), and the south-crest peak heights $(h m F 2)$ were about the same as the north-crest peak heights at $\sim 350 \mathrm{~km}$. Using the reference occultation data within 0200 to 0900 UT on Feb. 8, 1997, Fig. 5 shows four reconstructed 100-minute electron density images after 10 iterations of MART with relaxation parameters $\leq 0.01$ for each of the LEO-orbital revolutions. We note that the reconstructed images of Fig. 5, especially the two lower panels, show rougher shapes with no typical troughs of the equatorial anomaly feature as compared to the "true" ionospheric images of Fig. 3. Using the same data set but combining occultation observations in two and four LEO orbital revolutions, Figures 6 and 7 present the reconstructions for the two cases, respectively. Comparison of the equatorial anomaly features such as the crests and troughs in Figs. 6 and 7 with those shown in Fig. 3 shows quite similar results and better agreement between the reconstructed images and true IRI-modelled ionosphere. The LEO-orbital-plane latitudes and altitudes of south- and north-crests match fairly well, occurring at roughly +22 and -5 degree latitudes and $350 \mathrm{~km}$ altitude. The solpes of the lobes also match well. The peak density of the reconstruction having about 11.0 unit (each unit is $10^{11} \mathrm{el} \mathrm{m}^{-3}$ ), is slightly less than the peak densities shown in Fig. 3. The depression over the troughs shown in Figs. 6 and 7 has electron density between 6 and 8 units similar to those of Fig. 3 .

To further compare statistically the three cases of tomographic reconstructions, we have processed all occultation data in the same manner as described above (more than thirty-five hundred occultation records during 232 LEO-orbital revolutions) within 22 days of the 13th prime time (from Feb. 2 to Feb. 23 in 1997) of the GPS/MET mission and compared the results with the "true" IRI-modelled data. Three root-mean-square (RMS) electron density error images shown in Fig. 8 describe the accuracy of the reconstructed ionosphere. As is commonly the case, higher RMS error occurs where the electron density value is stronger. The error images therefore show similar fountain features as the equatorial anomaly. The results show the improvement of accuracy by combining occultation observations within two or four LEO orbital revolutions as compared to the case where data in only one revolution is used. This 

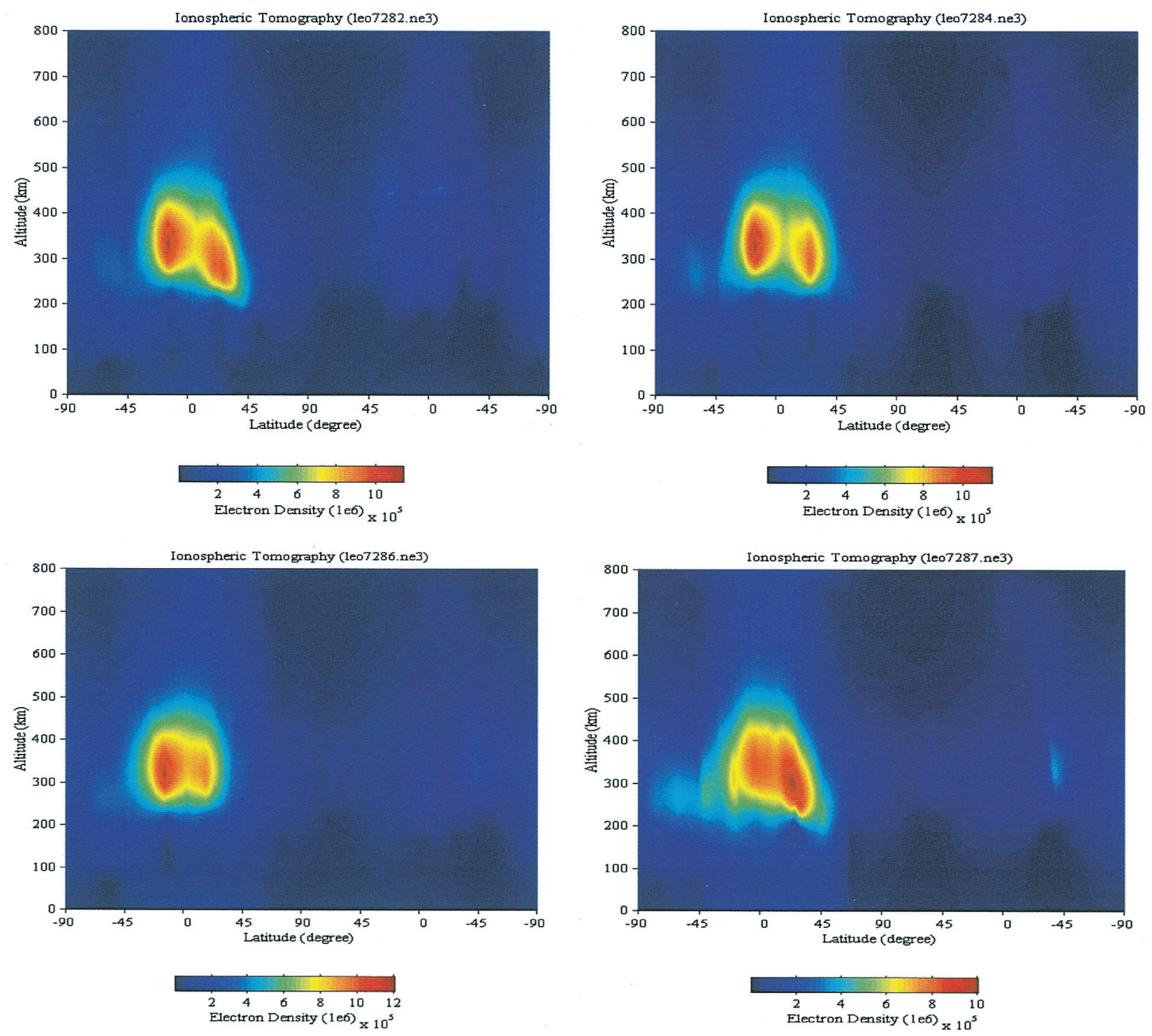

Fig. 5. Four contiguous 100-minute electron density images show ionospheric tomography inversed by the MART algorithm on simulated occultation links taking place during the same time as Fig. 3 within the reference GPS/MET experiment. Each tomography uses $\sim 17$ simulated occultation observation data recorded during one revolution of the MicroLab-1 satellite around the Earth.

seems to conclude that satisfactory reconstructions can be obtained from data combining occultation observations within two LEO orbital revolutions. 

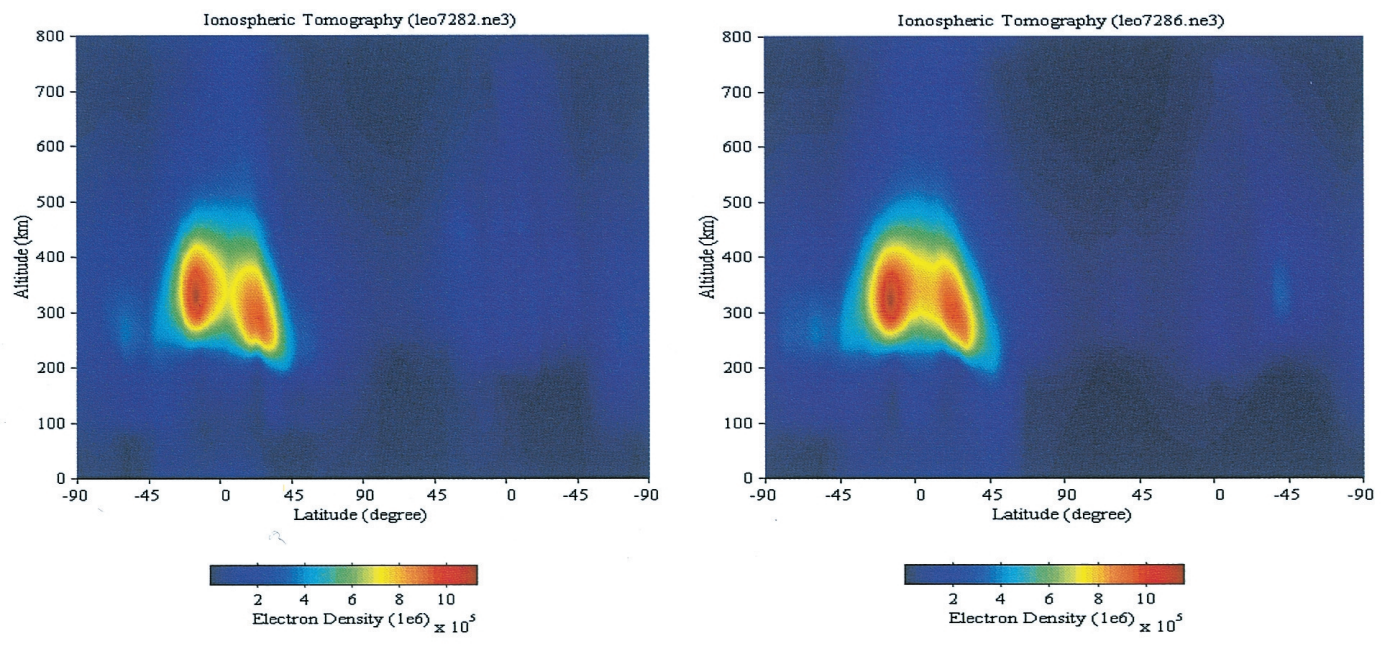

Fig. 6. Using the same occultation data as used for Fig. 5, two contiguous electron density images but each tomography uses twice of $\sim 17$ simulated occultation observation data recorded during two revolutions of the MicroLab-1 satellite around the Earth.

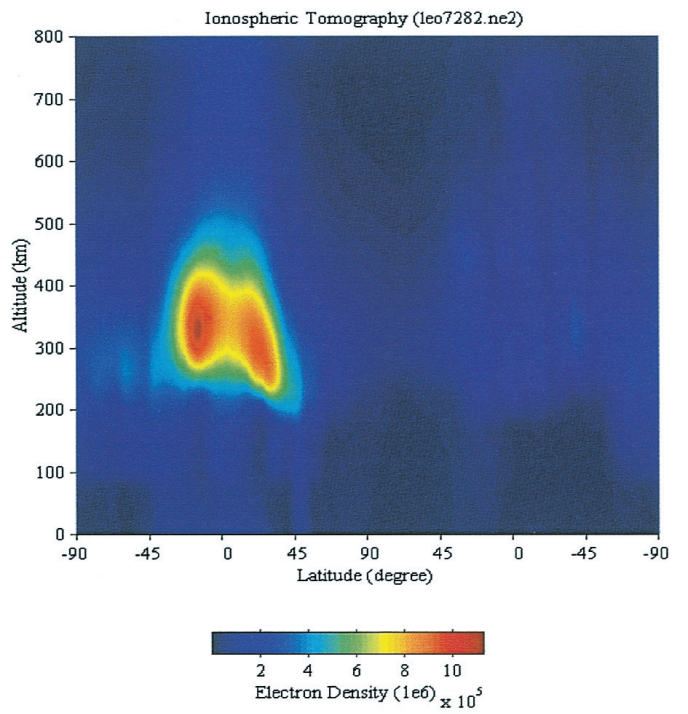

Fig. 7. Using the same occultation data as used for Fig. 5, the reconstructed tomography uses four of $\sim 17$ simulated occultation observation data recorded during four revolutions of the MicroLab-1 satellite around the Earth. 

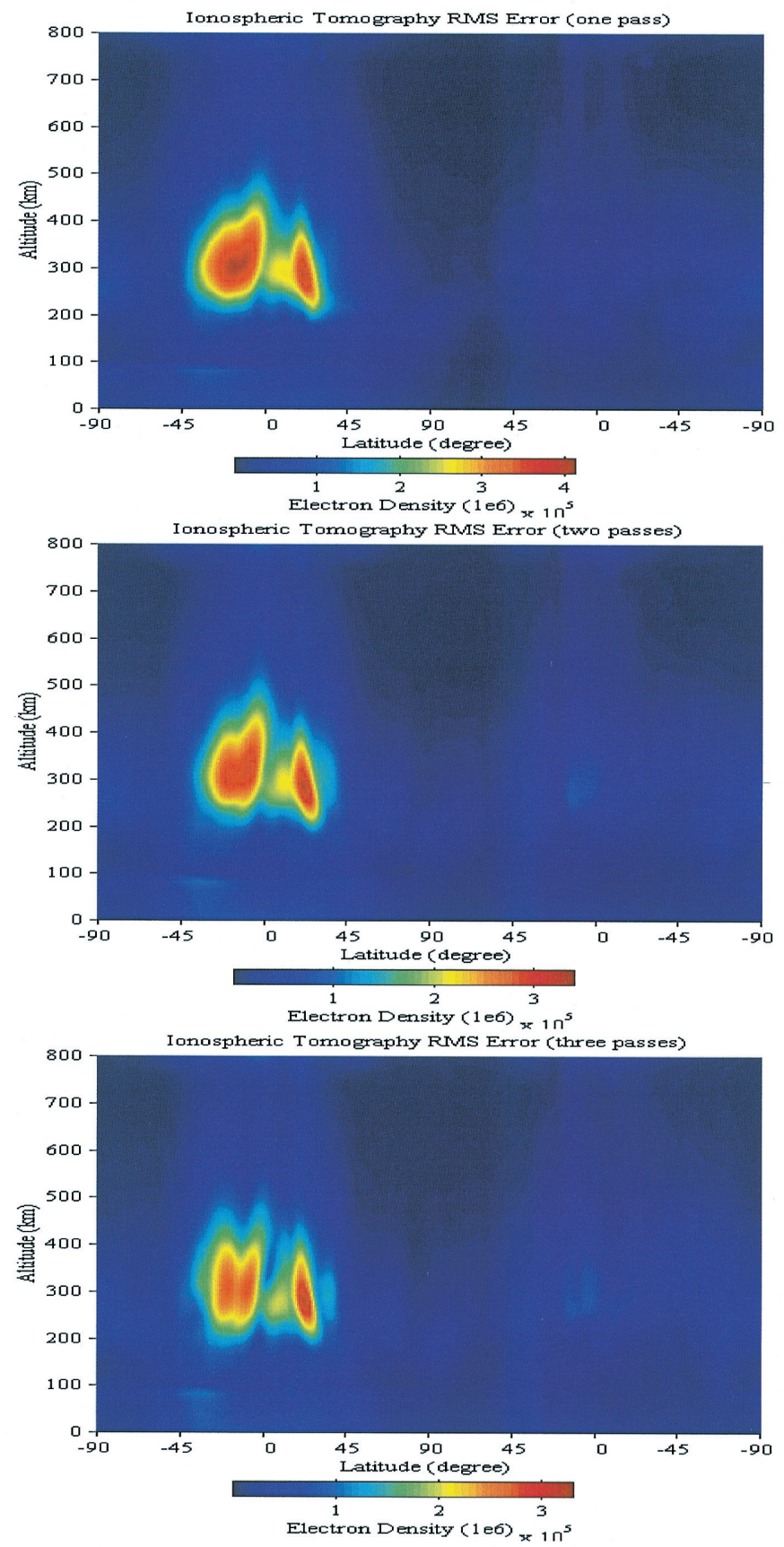

Fig. 8. RMS electron density error images for reconstructed tomographies using occultation observations within each one (upper), two (center), and four (lower) LEO orbital revolutions in the reference GPS/MET experiment. 


\section{CONCLUSIONS}

This paper discusses the feasibility of a space-based ionospheric tomography using the MART technique without additional data gained from ground-based observations. Instead, the electron density profiles derived from the GPS/MET type occultation experiment are used as the initial guess to start the iteration in MART. In our study, the data used in the reconstruction was based on simulated TEC data provide by the IRI-2001 model. The results of three test cases using combined reference GPS/MET data within one, two, and four passes of the LEO satellite orbit show convincingly improved equatorial anomaly features with improved agreement between the original IRI-modelled images and the tomographic reconstructions. It is as expected that increasing the number of occultation observations by combining observations within several orbital revolutions of the LEO satellite would increase the accuracy of reconstructed tomography but thus trade off temporal and/or spatial resolutions. In future work, placing one more occultation antenna for the space-based GPS receivers on the LEO satellite as planned in the Constellation Observing System for Meteorology Ionosphere and Climate (COSMIC) mission, could provide twice the number of occultation observations to determine more accurate tomographic electron densities. Tomographic investigations of this kind would give highly valuable information over large regions of the world especially ocean regions where no dense networks for ionospheric observations exist.

Acknowledgements We are grateful to Dr. W. S. Schreiner and D. C. Hunt (UCAR) who provided the $2^{\text {nd }}$ level ionospheric data of the GPS/MET experiment. This work has been supported by Projects NSC92-NSPO(B)-RS3-FA07-01D and NSC91-2111-M008-033.

\section{REFERENCES}

Austen, J. R., S. J. Franke, C. H. Liu, and K. C. Yeh, 1986: Application of computerized tomography techniques to ionospheric research. Proc. of the URSI and COSPAR International Beacon Satellite Symposium on Radio Beacon Contribution to the study of Ionization and Dynamics of the Ionosphere and to Corrections to Geodesy and Technical Workshop, part1, Univ. of Oulu, Finland, 23-35.

Austen, J. R., S. J. Franke, and C. H. Liu, 1988: Ionospheric imaging using computerized tomography. Radio Sci., 23, 299-307.

Bilitza, D., 1990: International Reference Ionosphere 1990, NSSDC 90-22, Greenbelt, Maryland.

Bilitza, D., 2001: International Reference Ionosphere 2000. Radio Sci., 36, 261-275.

Hajj, G. A., and L. J. Romans, 1998: Ionospheric electron density profiles obtained with the Global Positioning System: Results from the GPS/MET experiment. Radio Sci., 33, 175-190.

Hajj, G. A., L. C. Lee, X. Pi, L. J. Romans, W. S. Schreiner, P. R. Straus, and C. Wang, 2000: COSMIC GPS ionospheric sensing and space weather. Terr. Atmos. Ocean. Sci., 11, 235-272. 
Huang, C. R., C. H. Liu, H. C. Yeh, and W. H. Tsai, 1997: The low-latitude ionospheric tomography network (LITN) - initial results. J. Atmos. Terr. Phys., 59, 1553-1567.

Huang, C. R., C. H. Liu, K. C. Yeh, K. H. Lin, W. H. Tsai, H. C. Yeh, and J. Y. Liu, 1999: A study of tomographically reconstructed ionospheric images during a solar eclipse. $J$. Geophys. Res., 104, 79-94.

Kursinski, E. R., G. A. Hajj, J. T. Schofield, R. P. Linfield, and K. R. Hardy, 1997: Observing Earth's atmosphere with radio occultation measurements using the Global Positioning System. J. Geophys. Res., 102, 23429-23465, .

Lee, H., and G. Wade, 1985: Imaging Technology, IEEE Press, Yew York.

Rawer, K., D. Bilitza, and S. Ramakrishnan, 1978: Goals and status of the International Reference Ionosphere. Rev. Geophys., 16, 177-181.

Rawer, K., S. Ramakrishnan, and D. Bilitza, 1978: International Reference Ionosphere 1978, International Union of Radio Science, URSI Special report, Bruxells, Belgium, 75 pp.

Rawer, K., J. V. Lincoln, and R. O. Conkright, 1981: International Reference Ionosphere-IRI 79, World Data Center A for Solar-Terrestrial Physics, Report UAG-82, Boulder, Colorado, $245 \mathrm{pp}$.

Raymund, T. D., J. R. Austen, S. J. Franke, C. H. Liu, J. A. Klobuchar, and J. Sralker, 1990: Application of computerized tomography to the investigation of ionospheric structures. Radio Sci., 25, 771-789.

Raymund, T. D., S. J. Franke, and K. C. Yeh, 1994: Ionospheric tomograph: its limitations and reconstruction methods. J. Atmos. Terr. Phys., 56, 637-657.

Rius, A., G. Ruffini, and L. Cucurull, 1997: Improving the vertical resolution of ionospheric tomography with GPS occultations. Geophys. Res. Lett., 24, 2291-2294.

Rius, A., G. Ruffini, and A. Romeo, 1998: Analysis of ionospheric electron density distribution from GPS/MET occultations. IEEE Trans. Geosci. Remote Sens., 36, 383-394.

Schreiner, W. S., S. V. Sokolovskiy, C. Rocken, and D. C. Hunt, 1999: Analysis and validation of GPS/MET radio occultation data in the ionosphere. Radio Sci., 34, 949-966.

Sutton, E., and Helen Na, 1995: Comparison of geometries for ionospheric tomography. Radio Sci., 30, 115-125.

Tsai, L. C., W. H. Tsai, W. S. Schreiner, F. T. Berkey, and J. Y. Liu, 2001: Comparisons of GPS/MET retrieved ionospheric electron density and ground based ionosonde data. Earth Planets Space, 53, 193-205.

Tsai, L. C., C. H. Liu, W. H. Tsai, and C. T. Liu, 2002: Tomographic imaging of the ionosphere using the GPS/MET and NNSS data. J. Atmos. Terr. Phys., 64, 2003-2011.

Tsai, L. C., and W. H. Tsai, 2004: Improvement of GPS/MET ionospheric profiling and validation with Chung-Li ionosonde measurements and the IRI.Terr. Atmos. Ocean. Sci., 15, 589-607.

Ware, R., and Coauthors, 1996: GPS sounding of the atmosphere from low Earth orbit: preliminary results. Bull. Am. Met. Soc., 77, 19-40.

Zou, X. Y., H. Kuo, and Y. R. Guo, 1995: Assimilation of atmospheric radio refractivity using a nonhydrostatic mesoscale mode. Mon. Wea. Rev., 123, 2229-2249. 